\title{
Infra-Red Stable Supersymmetry in Chern-Simons Theories with Matter and Quenched Disorder
}

\author{
H. Hamidian \\ March 20, 2018 \\ Department of Physics, Stockholm University, Box 6730, S-113 85 Stockholm, Sweden

\begin{abstract}
We study Abelian Chern-Simons field theories with matter fields and global $S U(N)$ symmetry in the presence of random weak quenched disorder. In the absence of disorder these theories possess $\mathcal{N}=2$ supersymmetric fixed points and $\mathcal{N}=1$ supersymmetric fixed lines in the infra-red limit. We show that although the presence of disorder forbids any supersymmetry of the bare action, infra-red stable supersymmetric fixed points and fixed lines are realized in the disorder-averaged effective theories.
\end{abstract}

PACS number(s): 11.15.-q, 11.10.Kk, 11.30.Pb, 11.10.Hi, 11.30.Qc 
Model systems in less than four (space-time) dimensions have often played an important role in understanding general methods and applications of quantum field theory and developing ideas in four dimensions.

Notable among these are Chern-Simons (CS) gauge field theories in $(2+1)$ dimensions [1], which have a long history and have been intensively studied in recent years. There are several motivations for studying CS theories. From a purely theoretical and mathematical point of view they constitute a new class of (topological) gauge theories and are closely related to certain models in topological quantum field theory [2] in three space-time dimensions and integrable statistical mechanical models and rational conformal field theories in two dimensions [3]. From the more physical point of view there has been general interest in CS theories due to their resemblance to the high-temperature limit of $(3+1)$ dimensional gauge theories and their possible relevance to certain condensed matter phenomena, in particular high- $T_{C}$ superconductivity [4] and the quantum Hall effect [5].

Although CS gauge theories are in many respects similar to their four-dimensional counterparts, there are substantial differences which are intimately connected with the appearance of the CS three-form in the action [6] and parity anomalies [7]. Formally, CS gauge theories are strictly renormalizable quantum field theories and their perturbative expansions contain logarithmic divergences. However, contrary to the Yang-Mills and Maxwell gauge theories, and due to their topological nature, infinite charge renormalizations are absent in both Abelian and non-Abelian CS gauge theories [8, 9].

The situation changes significantly when matter fields are added to the CS theory. Although the CS gauge coupling remains unrenormalized as before, the matter couplings require renormalization. This leads to non-vanishing $\beta$-functions exhibiting a complicated and non-trivial renormalization group ( $\mathrm{RG}$ ) flow and a rich set of fixed points [10. By analyzing the CS gauge theory with matter couplings in the infrared (IR) limit the authors of Ref. [10] were able to show that, quite unexpectedly, some of the resulting fixed points and fixed lines exhibit $\mathcal{N}=1$ and $\mathcal{N}=2$ supersymmetry, which are realized as IR stable or saddle point solutions of the corresponding RG equations.

In this letter we study Abelian CS field theories with matter in the presence of random weak quenched disorder (QD) at zero temperature. The model is described by the (Euclidean) action,

$$
\begin{aligned}
S[\delta r]= & \int d^{3} x\left[\frac{i}{2} \epsilon^{\mu \nu \lambda} A_{\mu} \partial_{\nu} A_{\lambda}+\left|D_{\mu} \phi_{j}\right|^{2}+i \bar{\psi}_{j} \not D \psi_{j}\right. \\
& +\left(m^{2}+\delta r(x)\right) \phi_{j}^{*} \phi_{j}-M \bar{\psi}_{j} \psi_{j} \\
& -q\left(\phi_{j}^{*} \phi_{j}\right)^{2}-\alpha \bar{\psi}_{j} \psi_{j} \phi_{k}^{*} \phi_{k}-\beta \bar{\psi}_{j} \psi_{k} \phi_{j}^{*} \phi_{k} \\
& \left.-\frac{1}{4} \eta\left(\bar{\psi}_{j} \psi_{k}^{*} \phi_{j} \phi_{k}+\bar{\psi}_{j}^{*} \psi_{k} \phi_{j}^{*} \phi_{k}^{*}\right)+h\left(\phi_{j}^{*} \phi_{j}\right)^{3}\right],
\end{aligned}
$$

where $D_{\mu}=\partial_{\mu}-i e A_{\mu}$ is the covariant derivative and summation over repeated indices is implicitly assumed. Our notation and conventions are those of [10, 11]. The matter fields are in the fundamental representation of the global $S U(N)$ symmetry group $(j, k=1, \ldots, N)$ and the spinors are defined in the Majorana basis and under charge conjugation $C$ transform according to $C: \psi \rightarrow \psi^{*}$ [Note that Yukawa type vertices, which are in general allowed in 
three dimensions, are excluded from (1) since they violate fermion-number conservation]. The QD is described by random fluctuations of the disorder (field) $\delta r(x)$ whose probability distribution is taken to be symmetric and Gaussian:

$$
P[\delta r(x)]=p_{0} \exp \left\{-\frac{1}{4 \Delta} \int d^{3} x[\delta r(x)]^{2}\right\}
$$

with average fluctuations of width $\Delta \ll 1$,

$$
\left\langle\delta r(x) \delta r\left(x^{\prime}\right)\right\rangle_{P[\delta r]}=\Delta \delta^{3}\left(x-x^{\prime}\right)
$$

and $p_{0}$ the normalization constant.

In the absence of $\mathrm{QD}$, i.e. when $\delta r(x)=0$ in (1), and for the particular choice of the couplings,

$$
\begin{aligned}
& \alpha=\lambda, \beta=\lambda+e^{2}, \eta=2\left(\lambda-e^{2}\right), \\
& h=\lambda^{2}, m=M, q=2 \lambda m
\end{aligned}
$$

the theory possesses $\mathcal{N}=1$ and, upon imposing the further restriction $\lambda=e^{2}, \mathcal{N}=2$ supersymmetry; both of which are preserved by renormalization. By examining the RG equations for this theory the authours of Ref. [10] were able to establish the existence of $\mathcal{N}=1$ and $\mathcal{N}=2$ supersymmetric IR fixed points. The $\mathcal{N}=2$ supersymmetric fixed ponit exists for any choice of the global $S U(N)$ group. For $N=1$ the fixed point is IR stable and the critical exponents are Gaussian, while for $N>1$ the fixed points are saddle point solutions of the $\mathrm{RG}$ equations. The $\mathcal{N}=1$ supersymmetry manifests itself as a fixed line and is also valid for any value of $N$. The only fixed point on this line is the $\mathcal{N}=2$ supersymmetric one and is IR stable along the line, i.e. when the $\mathcal{N}=1$ supersymmetry is imposed.

The oiginal motivation that led us to consider the model described by (11) was to examine whether it is possible to modify the Abelian CS theory in such a way that the bare action would no longer support supersymmetry but, in the IR limit, the effective theory would flow towards stable supersymmetric fixed points and fixed lines as in the CS theory without QD. As will be discussed below, the inclusion of weak QD does indeed lead to such a possibility and could, in fact, arise in certain physically interesting theories. Among other applications, as mentioned above, the CS field theories have been considered as effective long-range theories of anyon superconductivity. In this context the CS fields arise from the Hopf term of an associated non-linear $\sigma$ model which describes spin excitations in doped quantum antiferromagnets [12] and it has been argued [4, 12] that a phase transition could occur which would lead either to superfluidity or, when the Maxwell field is introduced, to superconductivity. In studying such phase transitions the sytems are usually assumed to be perfectly homogeneous. However, in real samples defects and impurities are always present and can lead to different (critical) behavior. One can analyze the effect of impurities by using a field-theoretical description of phase transitions based on an effective Landau-Ginzburg formulation by introducing weak QD as, e.g., has been done here for the Abelian CS theory with matter. In other contexts, it may be possible to speculate on the role played by 
(topological) defects such as monopoles and strings in higher-dimensional theories, as long as these defects are sufficiently heavy and diluted and can be considered as effectively forzen-in on the scale of the entire system. In this case also one could imagine the system as described by an effective Landau-Ginzburg theory with weak QD which resembles the model studied here.

We shall now proceed to study the model described by (11) and explore whether there exist supersymmetric IR fixed points in the presence of weak QD and, if there are such fixed points, whether they are stable against QD.

In order to understand the effect of disorder one often invokes the so-called Harris citerion 13. According to this criterion, a change in the critical behavior due to disorder can occur only if the specific heat exponent $\alpha$ of the theory without disorder (the pure system) is positive. The physical reasoning which leads to this criterion is as follows: In the familiar case of theories with only a single mass parameter (e.g. the Landau-Ginzburg theory describing the 3D Ising model) criticality sets in at $T=T_{C}$, where one identifies the temperature with the mass term in the Lagrangian as $m_{\text {bare }}^{2} \sim t=\left(T-T_{C}\right) / T_{C}$. At the critical point the (renormalized) mass term vanishes and the correlation length $\xi \sim m^{-1}$ diverges like $\left(T-T_{C}\right)^{-\nu}$, for some exponent $\nu$. Writing $\Delta T \equiv T-T_{C} \sim \xi^{-1 / \nu}$ and noting that the (statistical) fluctuations $\delta T_{C}$ in $T_{C}$ due to QD obey $\delta T_{C} \sim \xi^{-D / 2}$, Harris has argued [13 that a sharp (second-order) transition is consistent if and only if the fluctuations in $T_{C}$ approach zero faster than does $\Delta T$ as $\xi$ approaches infinity. This occurs only when $\delta T_{C} / \Delta T=$ $\xi^{(1 / \nu-D / 2)} \rightarrow 0$ as $\xi \rightarrow \infty$, or $2-\nu D<0$. In other words, when $\alpha=2-\nu D<0$ the QD does not affect the critical behavior and one expects only second-order phase transitions (although treatments using the $\epsilon$-expansion suggest that IR stability is, in general, endangered by longrange quantum fluctuations [15]), whereas $\alpha>0$ indicates that including QD may either lead to (possibly new) IR stable fixed points or drive a smeared (first-order) phase transition.

As a first step to examine the effect of disorder on the critical behavior in the manner described above let us consider the critical exponent $\nu$ in the absence of QD which is given by the well-known relation [14 $\nu^{-1}=2-\gamma_{\phi^{2}}\left(g^{*}\right)$, where $\gamma_{\phi^{2}}\left(g^{*}\right)$ is the anomalous dimension of the $\phi^{2}$ operator at the fixed point $g^{*}$. The anomalous dimension at the critical point $g^{*}$ can be computed by using the RG equations for the Abelian CS theory without QD [10] and vanishes as $g \rightarrow g^{*}$ in the IR limit. [Note that it is always possible to renormalize at zero momentum in any dimension $d \leq 4$ and define renormalized $n$-point correlation functions, $\Gamma_{\mathrm{r}}^{(n)}\left(p ; m_{\mathrm{r}}, g_{\mathrm{r}}\right)=m_{\mathrm{r}}^{2}+p^{2}$, whose zero-momentum limit is relevant to the study of scaling behavior at criticality.] One therefore obtains $\alpha=1 / 2$ for the Abelian CS field theory with matter (and without QD) which, depending on the value of $\mathcal{N}$, corresponds to a Gaussian fixed point or fixed line. Since $\alpha>0$ one may expect the QD to somehow affect the critical behavior. However since the supersymmetric IR stable theory exhibits Gaussian critical behavior with $q=m=M=0$ at criticality, a closer examination is required. This is due to the existence of the additional mass parameters, $q$ and $M$, in the theory which lead to further conditions that have to be satisfied in order for the critical theory to be well-defined in the IR limit. In fact, although the dimensionless couplings in the theory simply approach constant values at the IR fixed points, the RG equations demand that both $q$ and $M$ vanish at the critical point. To see this it is sufficient to simply note that, as a result of the RG 
equations, the dimensionless quantities $\bar{q}=q / \mu$ and $\bar{M}=M / \mu$ ( $\mu$ is the RG scale) behave as

$$
\bar{q}^{2}(p) \sim q_{0}^{2} / p^{2}, \quad \bar{M}^{2}(p) \sim M_{0}^{2} / p^{2},
$$

to the leading order in the $p^{2} \rightarrow 0$ limit, which diverge unless also $q, M \rightarrow 0$ in the same limit. Therefore the low-energy (tri-)critical theory is consistently defined as long as not only $m$, but also $q$ and $M$ approach zero in the IR limit. By examining the $\beta$-functions in the theory without QD it can be easily confirmed that this is indeed the case [10]. By adding QD to the theory, as described by (1)-(3), a new dimensionful parameter, $\Delta$, is introduced which, on dimensional grounds, can be expected to affect the equations describing the RG flows of all the dimensionful couplings in the theory. Similarly to $\bar{q}$ and $\bar{M}$, by defining the dimensionless quantity $\bar{\Delta}=\Delta / \mu$ it follows that with the presence of QD a consistent (multi-)critical theory in the $p^{2} \rightarrow 0$ limit exists only if the QD parameter $\Delta$ (effectively) renormalizes to zero at the IR fixed point. To check whether this is realized and, if so, whether the IR fixed points remain stable against (quantum) fluctuations due to $\mathrm{QD}$ requires the knowledge of the $\mathrm{RG}$ equations that govern the flow towards the fixed points and fixed lines.

To derive the RG equations for the theory defined by (11) we must first compute the effective Landau-Ginzburg action by taking the average over the disorder $\delta r(x)$. This is most conveniently accomplished by using the standard replica trick [16] which enables one to compute the disorder-averaged effective action by using the identity $\ln Z=\lim _{n \rightarrow 0}\left(Z^{n}-1\right) / n$, where $Z$ is the partition function. Using the probability distribution (2), we obtain the (replica) action,

$$
\begin{aligned}
S_{Q D}= & \sum_{a=1}^{n} S_{a}[0]-\int d^{3} x \int d^{3} x^{\prime} \delta^{3}\left(x-x^{\prime}\right) \\
& \sum_{a, b=1}^{n} q_{a b}\left[\phi_{i}^{a}(x)\right]^{2}\left[\phi_{j}^{b}\left(x^{\prime}\right)\right]^{2} .
\end{aligned}
$$

Here $a, b=1, \ldots, n$ are the replica indices, $q_{a b}=q \delta_{a b}-\Delta$ and each replica $S_{a}[0]$ is of the same form as in (1) with the same replica index $a$ for all the fields. It is then straightforward to compute the RG equations by using the Feynman rules derived from (6) and taking the limit $n \rightarrow 0$. Since the theory is defined in three dimensions there are no genuine ultraviolet divergences in the one-loop approximation and the first non-trivial contributions arise from the two-loop diagrams. To this order, the $\beta$-functions which include the parameter $\Delta$ are

$$
\begin{aligned}
\beta_{\Delta}= & \left\{\frac{1}{3}\left[20 N \alpha^{2}+40 \alpha \beta+4(2 N+3) \beta^{2}+(2 N+5) \eta^{2}-40(N+2) e^{4}\right]+24(N+2) h\right\} \Delta, \\
\beta_{m^{2}}= & \frac{1}{3}\left[4\left(N \alpha^{2}+2 \alpha \beta+N \beta^{2}\right)+(N+1) \eta^{2}-4(5 N+4) e^{4}\right] m^{2} \\
& -\left[4\left(N \alpha^{2}+2 \alpha \beta+N \beta^{2}\right)+(N+1) \eta^{2}-4 N e^{4}\right] M^{2} \\
& +4(N+1)(q-\Delta)^{2}+4 N \Delta(q-\Delta),
\end{aligned}
$$

where $\beta_{\Delta}=\mu^{2} d \Delta / d \mu^{2}, \beta_{m^{2}}=\mu^{2} d m^{2} / d \mu^{2}$, and we have omitted the familiar factors of $\left(64 \pi^{2}\right)^{-1}$. The $\beta$-functions for the dimensionless couplings, $e, \alpha, \beta, \eta$ and $h$ and the dimen- 
sionful parameters $q$ and $M$ are not affected by QD to the order considered here and their corresponding expressions, which are somewhat lengthy, can be found in [10].

The fixed points correspond to the zeros of the $\beta$-functions for the dimensionless couplings. The CS gauge coupling $e$ has a vanishing $\beta$-function to all orders in perturbation theory and can be taken as a fixed parameter, while the other couplings become proportional to powers of $e$ with coefficients that are functions of $N$. Using the $\beta$-functions in (]) we find that the IR stable fixed points occur at $\Delta=0$. In particular, this implies that although the theory defined by (11) cannot support supersymmetry, the weak QD vanishes as the the IR stable $\mathcal{N}=1$ and $\mathcal{N}=2$ supersymmetric fixed lines and fixed points are approached and the Gaussian critical behavior is recovered. This non-trivial result provides an example for the existence of gauge theories with matter in which the (bare) Lagrangian does not possess supersymmetry but the theory flows towards a stable supersymmetric fixed point in the IR limit. Although we shall not present it here, we have checked that the same results are also true for certain non-Abelian CS field theories with matter. Non-Abelian CS field theories with matter with $S U(n), S p(n)$ and $S O(n)$ gauge groups were studied in [17] where similar behavior to the Abelian CS field theories were obtained. Adding QD to such theories leads to the same conclusions as for the Abelian theory discussed here. Although the Abelian and the non-Abelian theories exhibit quite different features - e.g. with respect to the dependence of the IR stability of the fixed points on the parameter $N$ of the global $S U(N)$ group - they both behave similarly with regard to the restoration of supersymmetry at the IR stable fixed points when QD is present.

To conclude, in this letter we have studied Abelian CS field theories with matter in the presence of random weak QD. We have shown that although in the presence of disorder these theories cannot support any supersymmetry of the bare action (unlike such theories without QD), stable supersymmetric fixed points are realized in the infra-red limit of the disorder-averaged effective theories. Several issues need to be explored in connection with this non-trivial behavior in three dimensions. An important phenomenological question that arises in the study of supersymmetric theories is to understand the origin of supersymmetry breaking. There are three main approaches to answer this question, namely: the introduction of explicit soft supersymmetry breaking terms, supersymmetry breaking in a hidden sector (such as the one based on $\mathcal{N}=1$ supergravity with $F$ and $D$ components of some chiral and vector superfields obtaining vev's at typically high energy scales) and supersymmetry breaking at low energies through interactions with the gauge fields [18]. All these approaches, however, still suffer from important drawbacks (see Dine in Ref. [18] for a brief review and further references) and there is much room for exploring other possibilities. In the three-dimensional model presented in this letter we have shown that it is possible to start with certain non-supersymmetric Lagrangians at some high energy scale, while maintainig supersymmetry in the low-energy effective theory. The supersymmetry of this effective theory can itself be broken by some appropriate mechanism to obtain another effective theory which is not supersymmetric. It is thus possible to imagine an intermediate supersymmetric theory between two non-supersymmetric theories in this way. The question of how many supersymmetric and non-supersymmetric fixed points are encountered at intermediate scales in the space of couplings before a desirable low-energy fixed point is reached is, of course, 
model dependent. To our knowledge, this type of behavior in supersymmetric theories has not been noticed prior to this work and whether similar behavior occurs in higher dimensional theories is an important question which could prove relevant to the general study of supersymmetry (breaking) in phenomenologically interesting models and deserves further investigation.

\section{ACKNOWLEDGEMENTS}

This work was supported by the Swedish Natural Science Research Council.

\section{References}

[1] R. Jackiw and S. Templeton, Phys. Rev. D 23, 2291 (1981); J. Schonfeld, Nucl. Phys. B185, 157 (1981); S. Deser, R. Jackiw, and S. Templeton, Ann. Phys. (NY) 140, 372 (1982), (E) 185, 406 (1988); Phys. Rev. Lett. 48, 975 (1982), (C) 59, 1981(1987).

[2] A.S. Schwartz, Lett. Math. Phys. 2, 247 (1987); Commun. Math. Phys. 67, 1 (1979); M.F. Atiyah, New invariants of three- and four-dimensional manifolds, in The Mathematical Heritage of Herman Weyl, Proc. Symp. Pure Math. 48, ed. R. Wells (Amer. Math. Society, Providence, RI, 1988); E. Witten, Commun. Math. Phys. 121, 351 (1989).

[3] G. Moore and N. Seiberg, in Physics, Geometry and Topology, Proceedings of Banff Summer School in Particles and Fields, Banff, Canada, ed. H.C. Lee (Plenum, New York, 1990), p.263.

[4] X.G. Wen and A. Zee, Phys. Rev. B 41, 240 (1990); Y.H. Chen, B.I. Halperin, F. Wilczek, and E. Witten, Int. J. Mod. Phys. B3, 1001(1989).

[5] S. Girvin, in The Quantum Hall Effect, ed. E. Prange and S.M. Girvin (Springer-Verlag, Berlin, 1986)p.380; B. Block and X.G. Wen, Phys. Rev. B 42, 8133, 8145 (1990).

[6] W. Siegel, Nucl. Phys. B156, 135 (1979); see also work cited in [1].

[7] A. Niemi and G. Semenoff, Phys. Rev. Lett. 51, 2077 (1983), Phys. Rep. 135, 3 (1987); N. Redlich, Phys. Rev. D 29, 2366 (1984); G.P. Korchemsky, Mod. Phys. Lett. A6, 727 (1991).

[8] S. Coleman and B. Hill, Phys. Lett. B 159, 184 (1985); G. Semenoff, P. Sodano, and Yong-Shi Wu Phys. Rev. Lett. 62, 715 (1989).

[9] E. Guadagnini, M. Martinelli and M. Mintchev, Nucl. Phys. B336, 581 (1990); A. Blasi and R. Collina, Nucl. Phys. B345, 472 (1990); F. Delduc, C. Lucchesi, O. Piguet, and S.P. Sorella, Nucl. Phys. 346, 313 (1990).

[10] G.V. Grigoryev and D.I. Kazakov, Phys. Lett. B 253, 411 (1991); L.V. Avdeev, G.V. Grigoryev, and D.I. Kazakov, Nucl. Phys. B382, 561 (1992). 
[11] S.J. Gates, M.T. Grisaru, M. Rocek, and W. Siegel, Superspace (Benjamin \& Cummings, Reading, MA, 1983).

[12] X.G. Wen and A. Zee, Phys. Rev. Lett. 63, 461 (1989), Nucl. Phys. B326, 619 (1989).

[13] A.B. Harris, J. Phys C7, 1671(1974); A.B. Harris and T.C. Lubensky, Phys. Rev. Lett. 33, 1540 (1974).

[14] J. Zinn-Justin, Quantum Field Theory and Critical Phenomena (New York, Oxford University Press, 1993).

[15] H. Hamidian, Phys. Lett. B 403, 108 (1997).

[16] S.F. Edwards in Polymer Networks, edited by A.J. Chompff and S. Newman (New York, Plenum Press, 1971); P.G. De Gennes, Phys. Lett. A 38, 339 (1972); G. Grinstein and A. Luther, Phys. Rev. B 13, 1329 (1976); V.J. Emery, Phys. Rev. B 11, 239 (1975); S.F. Edwards and P.W. Anderson, J. Phys. F5, 965 (1976).

[17] L.V. Avdeev, D.I. Kazakov and I.N. Kondrashuk, Nucl. Phys. B391, 333 (1993).

[18] S. Dimopoulos and H. Georgi, Nucl. Phys. B193, 150 (1981); H.P. Nilles, Phys. Repts. 110, 1 (1984); M. Dine, Nucl. Phys. B (Proc. Suppl.) 52A, 201 (1997). 